\title{
A NOTE ON M/G/1 VACATION SYSTEMS WITH SOJOURN TIME LIMITS
}

\author{
TSUYOSHI KATAYAMA, * Chubu Teletraffic Engineering Laboratory
}

\begin{abstract}
In this paper we deal with an M/G/1 vacation system with the sojourn time (wait plus service) limit and two typical vacation rules, i.e. multiple and single vacation rules. Using the level crossing approach, we derive recursive equations for the steady-state distributions of the virtual waiting times in $\mathrm{M} / \mathrm{G} / 1$ vacation systems with a general vacation time and two vacation rules.
\end{abstract}

Keywords: Sojourn time limit; vacation system; balking; level crossing; integral equation

2010 Mathematics Subject Classification: Primary 45D05; 60G17; 60K25

\section{Introduction}

We consider an M/G/1 vacation system with the sojourn time limit $(T)$, in which all customers without blocking at their arrival instants are guaranteed service completion as described below. For the M/G/1 vacation system, the following integral equation for the probability density function, $w(x)$, of the virtual waiting time $(W)$ was formulated in Katayama (2011) using the level crossing approach (see, e.g. Brill (2008)):

$$
w(x)= \begin{cases}\lambda_{v} \bar{V}(x)+\lambda W(0)\{\bar{H}(x)-\bar{H}(T)\} & \\ \quad+\lambda \int_{0+}^{x}\{\bar{H}(x-y)-\bar{H}(T-y)\} w(y) \mathrm{d} y, & T>x>0, \\ \lambda_{v} \bar{V}(x), & x \geq T .\end{cases}
$$

Here $\lambda$ and $\lambda_{v}$ are the arrival rates of ordinary and vacation customers, respectively. The service time of a customer $H$ and the vacation time of a vacation customer $V$ are independent and identically distributed random variables. Given a nonnegative-valued random variable $F$, we denote its distribution function (DF) by $F(t)$, its probability density function (PDF) by $f(t)$, its Laplace-Stieltjes transform by $F^{*}(s)$, and its finite first and $m$ th moments of the DF by $f$ and $f^{(m)}, m=2,3, \ldots$, respectively. We define $\bar{F}(t):=1-F(t)$ for $t \geq 0$, and $\rho:=\lambda \mathrm{E}(H)$ and $\rho_{v}:=\lambda_{v} \mathrm{E}(V)$.

The solution of the above integral equation for $w(x)$, however, has been given for only exponential service time and constant service time distributions, that is, the $M / M / 1$ and the $\mathrm{M} / \mathrm{D} / 1$ models. In the M/G/1 vacation system with sojourn time limit, customers are served by a single server in order of arrival (first-in-first-out (FIFO) discipline). They cannot stay in the system longer than an interval of length $T$. We assume that the sojourn times of arriving customers are known by a system manager at their arrival instants, and that they are refused entry to the system if their sojourn time $\Theta$ exceeds the interval $T$, i.e. $\Theta>T$. (The system

Received 29 November 2011; revision received 3 May 2012.

* Postal address: Chubu Teletraffic Engineering Laboratory, Naka-taikouyama 4-66, Imizu-shi, Toyama, 939-0363, Japan. Email address: katagon@ silk.plala.or.jp 
manager may utilize a reneging rule before starting service for the blocked customers.) If the queue becomes empty at the end of a service period, the server takes a vacation with vacation time $V$ (i.e. the so-called exhaustive service vacation).

We consider the following two typical vacation rules.

(i) If the system is empty upon the server's return from a vacation, another vacation begins immediately. If the system is not empty upon the server's return, the server starts servicing again (multiple vacation (MV) rule).

(ii) If the system is empty upon the server's return from a vacation, the server does not take another vacation and waits for a new arrival. If the system is not empty upon the server's return, the server starts servicing again, i.e. the server takes exactly one vacation (single vacation (SV) rule).

\section{Preliminaries}

We recall the virtual workload process $\left\{W_{t}\right\}$ of the M/G/1 vacation system with the sojourn time limit, which leads to (1) and (2), where $W_{t}$ represents the virtual waiting time $(W)$ at time $t \geq 0$ under the FIFO discipline; see Figures 1 and 2 of Katayama (2011), depicting typical sample paths for the MV and SV rules, respectively. The virtual workload process $\left\{W_{t}\right\}$ is a stochastic process with independent cycles defined as an interval between successive vacation starting epochs, in which the expected length of a cycle is denoted by $\mathrm{E}(C)$.

Next we prepare the following two propositions on the SV rule for the analysis of $W(0)$ in the next section. Let us denote by $I$ a nonbusy period immediately following a vacation, in which $W_{t}=0$. Furthermore, let us denote by $b(T: n \geq 1)$ the probability that only blocked customers, at least one customer, arrive during a vacation.

Proposition 1. The PDF of I, $i(x)$, for the SV rule and the first moment of I are given by

$$
i(x)=\lambda H(T) \mathrm{e}^{-\lambda H(T) x}
$$

and

$$
\mathrm{E}(I)=\frac{1}{\lambda H(T)} .
$$

Proof. Let $i_{n}(x) \mathrm{d} x$ denote the probability that only $n-1, n=1,2,3, \ldots$, blocked customers arrive successively before the $n$th customer, i.e. the first nonblocked customer, arrives at a small interval $(x, x+\mathrm{d} x)$. Then we obtain

$$
i_{n}(x) \mathrm{d} x=p_{n-1}(x) \lambda \mathrm{d} x \bar{H}(T)^{n-1} H(T) \quad \text { for } n \geq 1
$$

and

$$
p_{n}(x):=\frac{(\lambda x)^{n}}{n !} \mathrm{e}^{-\lambda x},
$$

where $\bar{H}(T)$ represents the probability that a customer is blocked at the arrival instant, because $W_{t}=0$. Hence, $\sum_{n=1}^{\infty} i_{n}(x)$ leads to $i(x)$ and $\mathrm{E}(I)$.

Proposition 2. The probability $b(T: n \geq 1)$ is expressed as

$$
b(T: n \geq 1)=\sum_{n=1}^{\infty} \int_{0}^{\infty} p_{n}(x) G_{n}(x, T) \mathrm{d} V(x),
$$


where

$$
\begin{aligned}
p_{n}(x) & :=\frac{(\lambda x)^{n}}{n !} \mathrm{e}^{-\lambda x}, \\
G_{n}(x, T) & :=\prod_{i=1}^{n} \int_{y_{i}} \frac{\bar{H}\left(T-x+y_{i}\right)}{x} \mathrm{~d} y_{i} \\
& =\frac{1}{x^{n}} \int_{y_{1}=0}^{x} \int_{y_{2}=y_{1}}^{x} \cdots \int_{y_{n}=y_{n-1}}^{x} \prod_{i=1}^{n} \bar{H}\left(T-x+y_{i}\right) \mathrm{d} y_{i},
\end{aligned}
$$

with $0<y_{1}<\cdots<y_{n-1}<y_{n} \leq x$ and $\bar{H}\left(T-x+y_{i}\right) \equiv 1$ for $T-x+y_{i} \leq 0$.

Proof. The function $p_{n}(x)$ represents the probability that $n$ customers, who arrive at $0<y_{1}<y_{2}<\cdots<y_{n-1}<y_{n} \leq x$, arrive during a vacation, given $V=x$. Note here that the arrival instants $U_{i}, i=1,2, \ldots, n$, are uniformly distributed in the interval $(0, x]$ under the condition that $n$ customers arrive during the vacation time $V=x$. For the customer that arrived at $U_{i}=y_{i}$, the probability that this is blocked, i.e. $\Theta=W+H>T$, is given by $\bar{H}\left(T-x+y_{i}\right)$, because $W_{t}(=W)$ equals $x-y_{i}$. That is, the probability that $n \geq 1$ customers arrive in the interval $(0, x]$ and all of the $n$ customers are blocked is given by $p_{n}(x) G_{n}(x, T)$. Hence, unconditioning of $p_{n}(x) G_{n}(x, T)$ leads to $b(T: n \geq 1)$. In the case where $T<V=x$, it possibly happens that $T-x+y_{i} \leq 0$. Then we have to put $\bar{H}\left(T-x+y_{i}\right) \equiv 1$.

\section{Virtual waiting time analyses for MV and SV rules}

From (1) and (2), we obtain the following results for a general service time distribution.

Theorem 1. For the $M / G / 1$ vacation systems ( $M V$ and $S V$ rules) with limited sojourn time $(T)$, the PDF of the virtual waiting time, $w(x), x>0$, and $W(0)$ are given by

$$
\begin{aligned}
w(x) & = \begin{cases}\lambda_{v} \omega_{0}(x), & x<T, \\
\lambda_{v} \bar{V}(x), & x \geq T,\end{cases} \\
\omega_{0}(x) & :=\varphi_{0}(x)+\sum_{n=1}^{\infty} \lambda^{n} \varphi_{n}(x), \\
\varphi_{n}(x) & :=\int_{0+}^{x} K(x, y) \varphi_{n-1}(y) \mathrm{d} y, \quad n=1,2,3, \ldots, \\
\varphi_{0}(x) & :=\bar{V}(x)+\lambda W^{+}(0)\{\bar{H}(x)-\bar{H}(T)\}, \\
K(x, y) & :=\bar{H}(x-y)-\bar{H}(T-y), \\
\lambda_{v} & :=\left(W^{+}(0)+\int_{0+}^{T} \omega_{0}(x) \mathrm{d} x+\int_{T}^{\infty} \bar{V}(x) \mathrm{d} x\right)^{-1}, \\
W(0) & =\lambda_{v} W^{+}(0),
\end{aligned}
$$

and

$$
W^{+}(0):= \begin{cases}0 & \text { for the MV rule, } \\ \frac{V^{*}(\lambda)+b(T: n \geq 1)}{\lambda H(T)} & \text { for the SV rule, }\end{cases}
$$

where the probability $b(T: n \geq 1)$ is given in (3). 
Proof. It follows from the observation of a sample path $W_{t}$ that $W(0)=0$ for the MV rule, and, for the SV rule,

$$
\begin{gathered}
\lambda_{v}=\frac{1}{\mathrm{E}(C)}, \\
W(0)=\frac{q_{0} \mathrm{E}(I)+b(T: n \geq 1) \mathrm{E}(I)+\left\{1-q_{0}-b(T: n \geq 1)\right\} \times 0}{\mathrm{E}(C)}, \\
\mathrm{E}(I)=\frac{1}{\lambda H(T)}, \quad q_{0}:=V^{*}(\lambda), \quad b(T: n \geq 1)=\sum_{n=1}^{\infty} \int_{0}^{\infty} p_{n}(x) G_{n}(x, T) \mathrm{d} V(x),
\end{gathered}
$$

using the property of the regenerative process $\left\{W_{t}\right\}$; see, e.g. Chapter VI of Asmussen (2003). We now comment on how to derive the numerator on the right-hand side of (9). The first term corresponds to the case where no customers arrive during a vacation and after the vacation only blocked customers arrive successively before a nonblocked customer arrives. The second term corresponds to the case where only blocked customers, at least one customer, arrive during a vacation and after the vacation only blocked customers arrive successively before a nonblocked customer arrives. For the other case, the mean nonbusy period is 0 , where $1-q_{0}-b(T: n \geq 1)$ in the last term represents the probability that nonblocked customers, at least one customer, arrive during a vacation. Hence, these facts lead to (7) and (8). For the MV and SV rules, (1) is rewritten as

$$
w(x)=\lambda_{v} \varphi_{0}(x)+\lambda \int_{0+}^{x} K(x, y) w(y) \mathrm{d} y,
$$

where $\varphi_{0}(x)$ and $K(x, y)$ are defined in (6). This integral equation is called a Volterra equation of the second kind. Now we introduce the series $\left\{f_{n}(x)\right\}$ satisfying the relation

$$
w(x)=\sum_{n=0}^{\infty} \lambda^{n} f_{n}(x), \quad T>x>0,
$$

which is also called a Neumann series; see Remark 1 below. Then, it follows from (1) that

$$
\begin{aligned}
\sum_{n=0}^{\infty} \lambda^{n} f_{n}(x) & =\lambda_{v} \varphi_{0}(x)+\sum_{n=0}^{\infty} \lambda^{n+1} \int_{0+}^{x} K(x, y) f_{n}(y) \mathrm{d} y \\
& =\lambda_{v} \varphi_{0}(x)+\sum_{n=1}^{\infty} \lambda^{n} \int_{0+}^{x} K(x, y) f_{n-1}(y) \mathrm{d} y
\end{aligned}
$$

Comparing the left-hand side with the right-hand side of (11), we obtain the recursive equations

$$
f_{n}(x)=\int_{0+}^{x} K(x, y) f_{n-1}(y) \mathrm{d} y, \quad n=1,2,3, \ldots, \quad f_{0}(x)=\lambda_{v} \varphi_{0}(x),
$$

that is,

$$
\varphi_{n}(x)=\int_{0+}^{x} K(x, y) \varphi_{n-1}(y) \mathrm{d} y, \quad f_{n}(x)=\lambda_{v} \varphi_{n}(x), \quad n=1,2,3, \ldots
$$

Hence, using (10) and (13), $w(x), T>x>0$, can be expressed as in (5) together with (6), where $\lambda_{v}$ is yet unknown. For $x \geq T, w(x)$ is given by (2), directly. The arrival rate $\lambda_{v}$ of 
vacation customers should be determined from the normalization condition,

$$
W(0)+\int_{0+}^{T} w(x) \mathrm{d} x+\int_{T}^{\infty} w(x) \mathrm{d} x=1,
$$

which concludes the proof.

Remark 1. It is known that the Volterra equation (1) with kernel $K(x, y)<\infty$ has a unique solution $w(x)$, given by (10), for every continuous function $\lambda_{v} \varphi_{0}(x)<\infty$ and every complex value of $|\lambda|<\infty$, which is ensured by the DFs $H(x)$ and $V(x)$ and the arrival rate $\lambda$ in the vacation systems analyzed in this section; see, e.g. Chapter VI of Roubine (1970). (The explicit solution (10) for a general service time distribution would be useful to discuss computational implications for $w(x)$. However, the formula $\sum_{n=0}^{N} \lambda^{n} f_{n}(x)$ together with (12) has an inevitable problem due to a truncated error given by $\sum_{n=N+1}^{\infty} \lambda^{n} f_{n}(x)$, i.e. the convergence speed to compute $\left\{f_{n}(x), n=1,2,3, \ldots\right\}$. Furthermore, a similar problem arises from computing (3) together with (4) for the SV rule.)

Using Theorem 1 and the PASTA (Poisson arrivals see time averages) property, the following performance measures for an arbitrary ordinary customer are obtained for the MV and SV rules. Let $p$ denote the blocking probability (or loss probability) that a customer cannot enter the system upon arrival, i.e. its sojourn time $\Theta>T$.

Corollary 1. The blocking probability in the $M / G / 1$ vacation system with limited sojourn time $T$ is given by

$$
p=\lambda_{v}\left(W^{+}(0) \bar{H}(T)+\int_{0+}^{T} \bar{H}(T-x) \omega_{0}(x) \mathrm{d} x+\int_{T}^{\infty} \bar{V}(x) \mathrm{d} x\right),
$$

where $\lambda_{v}, W^{+}(0)$, and $\omega_{0}(x)$ are given in (6) and (8) for both the MV and SV rules. The DF of the actual waiting time for a nonblocked customer is given by

$$
W(x \mid \text { no blocking })=\frac{\lambda_{v}}{1-p}\left(H(T) W^{+}(0)+\int_{0+}^{x} H(T-y) \omega_{0}(y) \mathrm{d} y\right), \quad T>x>0,
$$

where $\lambda_{v}, W^{+}(0)$, and $\omega_{0}(x)$ are given in (6) and (8) for both the MV and SV rules. The probability of no delay for a nonblocked customer is given by

$$
W(0 \mid \text { no blocking })= \begin{cases}0 & \text { for the } M V \text { rule, } \\ \frac{\lambda_{v}\left\{V^{*}(\lambda)+b(T: n \geq 1)\right\}}{\lambda(1-p)} & \text { for the SV rule, }\end{cases}
$$

where $b(T: n \geq 1)$ and $\lambda_{v}$ are given in (3) and (6), respectively. The mth moment, $m=1,2, \ldots$, of the actual waiting time is given by

$$
\mathrm{E}\left(W^{m} \mid \text { no blocking }\right)=\frac{\lambda_{v}}{1-p} \int_{0+}^{T} x^{m} H(T-x) \omega_{0}(x) \mathrm{d} x,
$$

where $\lambda_{v}$ and $\omega_{0}(x)$ are given in (6) and (8) for both the MV and SV rules.

Proof. By definition,

$$
p=\frac{1}{\lambda}\left(\lambda W(0) \bar{H}(T)+\lambda \int_{0+}^{T} \bar{H}(T-x) w(x) \mathrm{d} x+\lambda \int_{T}^{\infty} w(x) \mathrm{d} x\right),
$$


which leads to (14), and

$$
W(x \mid \text { no blocking })=\frac{1}{1-p} \int_{0}^{x} H(T-y) \mathrm{d} W(y),
$$

which leads to (15), (16), and (17).

Remark 2. The functions $W(x)$ and $w(x)$ exist even for $1 \leq \rho+\rho_{v}<\infty$, since the vacation systems analyzed in this section are a loss and delay system with the sojourn time limit for ordinary customers. (Such a result has been found for the dam model in Takács (1967).) The DF of the actual waiting time $W(x \mid$ no blocking), $T>x \geq 0$, is not affected by the reneging rule introduced in Section 1. Propositions 1 and 2, i.e. (9), should also be applied to Theorems 2 and 4 of Katayama (2011) for the SV rule, i.e. Equation (9) therein. The stochastic decomposition property exists in the vacation systems considered in the present paper; see, e.g. Fuhrmann and Cooper (1985). Similar sojourn time limit queueing models without service vacations have been studied in Gavish and Schweitzer (1977) for the M/M/1 model, in Hokstad (1979) for the M/D/1 model, and in Perry and Asmussen (1995) for the M/G/1 model (Model III) (note that Equations (2.8) and (2.11) of Perry and Asmussen (1995) contain typographical errors and use different notation to that used in (13)).

\section{References}

Asmussen, S. (2003). Applied Probability and Queues (Appl. Math. 51), 2nd edn. Springer, New York.

Brill, P. H. (2008). Level Crossing Methods in Stochastic Models (Internat. Ser. Operat. Res. Manag. Sci. 123). Springer, New York.

Fuhrmann, S. W. ANd CoOPER, R. B. (1985). Stochastic decompositions in the M/G/1 queue with generalized vacations. Operat. Res. 33, 1117-1129.

Gavish, B. And Schweitzer, P. J. (1977). The Markovian queue with bounded waiting time. Manag. Sci. 23, 13491357.

Hokstad, P. (1979). A single-server queue with constant service time and restricted accessibility. Manag. Sci. 25, 205-208.

Katayama, T. (2011). Some results for vacation systems with sojourn time limits. J. Appl. Prob. 48, $679-687$.

Perry, D. And Asmussen, S. (1995). Rejection rules in the M/G/1 queue. Queueing Systems 19, 105-130.

Roubine, É. (ed.) (1970). Mathematics Applied to Physics. Springer, New York.

TaKács, L. (1967). The distribution of the content of finite dams. J. Appl. Prob. 4, 151-161. 